\title{
IMPLANTATION OF RARE-EARTH ATOMS INTO Si AND III-V COMPOUNDS
}

\author{
A. Kozanecki, J.M. Langer \\ Institute of Physics, Polish Academy of Sciences \\ Al. Lotników 32/46, 02-668 Warszawa, Poland \\ AND A.R. PEaker \\ University of Manchester Institute of Science and Technology \\ P.O. Box 88, Sackville Str., Manchester, M60 1QD, United Kingdom \\ Dedicated to Professor Dr. Julian Auleytner \\ on the occasion of his 70 th birthday
}

(Received November 20, 1992)

\begin{abstract}
Most recent results on doping of Si and III-V semiconductors by the implantation of rare-earth atoms are reviewed. It is shown that up to the concentration of about $10^{18} \mathrm{~cm}^{-3}$ clustering and precipitation can be avoided. Post-implantation annealing leads not only to a decrease in radiation damage, but in some cases also to migration of rare-earth implants. The results of the rare earth lattice location by the Rutherford backscattering measurements are also reported.
\end{abstract}

PACS numbers: $61.80 . \mathrm{Jh}, 78.55 .-\mathrm{m}$

\section{Introductory remarks}

Recently we witness a burst of interest in the doping of Si and III-V compound semiconductors with rare earths (RE). The attention to these exotic dopants was attracted by their potential utilization in construction of novel light emitting devices (LEDs). Rare earth doped semiconductors are expected to combine the well-known sharp, temperature stable, ultra narrow, atomic-like emission originating from the electronic transitions within the $4 f$ shell of RE impurities with the efficient electrical pumping of this emission by electrons and holes. The inherently long lifetime of the $4 f$ emission limits a possible light output from these devices to small power in a microwatt range. However, if RE doping of Si would result in the construction of non-degrading efficient LEDs, even with a moderate quantum efficiency at room temperature, then this could lead to a real intrachip 
optical communication - a long awaited technological goal in the field of VLSI circuits $[1,2]$.

Rare earths are very often used as the light emitting centres in wide energy gap ionic crystals and glasses. In fact, most powerful solid state lasers are made of an Nd doped glass. REs are also often utilised in other solid state lasers. In all these cases high dopant concentration is achieved during the crystal or glass growth by adding required amounts of appropriate RE compound to the melt from which a laser rod is pulled out.

In semiconductor devices, the active volume is much smaller and planar technologies dominate processing. Very low solubility of REs in covalent semiconductors narrows the range of a vailable technologies of the growth of RE doped semiconductors to non-equilibrium techniques. Among them, two are feasible. One bases on metallo-organic chemical vapour deposition (MOCVD) - a standard technology for semiconductor growth. Initial problem of finding suitable-RE compound which would crack in the MOCVD reactor seems to be finally resolved. This technology has been mastered particularly by the group of $\mathrm{K}$. Takahei [3]. Alternative technology, most suitable for Si doping with REs, is ion implantation, which will be the main topic of our review.

Additional problem related to RE doping of $\mathrm{Si}$ and III-V compounds is caused by extremely high chemical activity of the RE elements, particularly their notorious affinity to oxygen and other group-VI elements. This can be turned into advantage, however, in the purification of the materials grown from the melt (i.e. liquid phase epitaxy) in the presence of REs [4]. Also, the tendency of association of RE atoms with other chemical dopants and lattice defects may have a beneficial effect on the increase in the excitation efficiency of the intrashell luminescence of REs in semiconductors.

Also the mismatch between the fourfold coordinated zinc-blende structure of semiconductors and the sixfold coordination of the RE atoms in stoichiometric compounds of lanthanides with $\mathrm{P}$ or $\mathrm{As}$ (cubic $\mathrm{NaCl}$ structure) may be another restriction on incorporating the RE ions into the substitutional positions in III-V crystal lattices. Moreover, it was quite recently shown [5] that RE-GaAs systems are thermodynamically unstable. Evaporation of RE metals on the GaAs surface in high vacuum causes dissociation of the $\mathrm{Ga}-\mathrm{As}$ bonds and subsequent formation of the $\mathrm{RE}$ arsenides. The melting points of $\mathrm{RE}$ arsenides are of the order of $2500^{\circ} \mathrm{C}$, therefore it can be expected that cubic complexes of the RE atoms with As, and $\mathrm{P}$ atoms, once formed, may be very resistant to decomposition during thermal treatment of the samples.

\section{Ion implantation doping and Rutherford backscattering}

Ion implantation is a convenient method of introducing dopants into semiconducting materials. The ion dose and ion energy, are well controlled, therefore the impurity and damage distributions in the materials are reliably calculated using standard computer codes, as e.g. TRIM [6]. Calculations using this code show that the projected range of the lanthanide atoms in semiconductors is less than $100 \mathrm{~nm}$ for typical implantation energies from 100 to $500 \mathrm{keV}$. A serious 
disadvantage of ion implantation is associated with extended damage produced by energetic heavy ions. In case of REs a dose of $10^{13} \mathrm{~cm}^{-2}$ is sufficient to amorphize completely the surface layers of semiconductors at room temperature (RT). Since, as a rule, the recrystallization of the layers is never complete in III-V compounds [7], ion implantation should be done at elevated temperatures.

As doping by implantation occurs near the surface, the simplest and most reliable method of determining the location of an atom (heavier than host crystal atoms) is by Rutherford backscattering (RBS) and channelling [8]. In the first approximation the ratio of the channelling/random backscattering yields gives an approximate interstitial fraction of the impurity atoms. A more detailed account of the RBS technique can be found e.g. in $[6,8]$.

\section{RE doping of silicon}

Silicon is the basic semiconductor material used in contemporary microelectronics. It has, however one significant drawback. Due to its indirect gap, the probability of optical transitions is very low and this is the reason for Si not being suitable for making light emitting devices. Despite many attempts to relax the momentum conservation rule for band-band related transitions, e.g. by doping (like in the case of GaP:N), the results were not satisfactory. One of the most promising alternative paths is the light generation from the intraimpurity transitions excited by the injected charge carriers. Rare earths are among those impurities that could provide such an emission. It is, however, well known that the diffusivity and solubility of these impurities is extremely low $[9,10]$. Moreover, REs have a tendency to form complexes with other defects in $\mathrm{Si}$, particularly oxygen and other group-VI impurities. Also they readily form silicides, and thus at higher concentrations precipitation of a new phase is expected (as it was observed in heavy doped GaSb [11]). In Er implanted Si the onset of Er precipitation is found to occur when $[\mathrm{Er}]>1.3 \times 10^{18} \mathrm{~cm}^{-3}$ for annealing temperature of $900^{\circ} \mathrm{C}$ [12]. A similar result has recently been obtained on molecular beam epitaxy (MBE) grown Si doped with Er. Here transmission electron microscopy measurements revealed onset of precipitation at the same Er concentration [13].

Most of attempts to dope Si with rare earths were done by ion implantation. Defects created by ion implantation in Si are known and well characterised, and what is even more important, the annealing procedures of the radiation damage are also well known. However, bonding of the rare earths to the host atoms may differ from the $s p^{3}$-type bonding. Therefore, the validity of extrapolation of the well-known post implantation procedures cannot be taken for granted. This is well exemplified by a recent controversy concerning the electrical activity of RE dopants introduced by implantation.

Rare earths should be acceptors if substitutional. This simple notion has been confirmed by the recent model calculations [14]. However, there are several reports claiming a donor type behaviour of RE implants [15, 16]. Although the concentration of these donors follows trends in an overall RE concentration determined by secondary-ion mass spectrometry, their absolute concentration is one to two orders of magnitude lower. It was therefore concluded that most of REs 
form electrically inactive complexes, while only a small portion form donors [16]. It is quite likely, however, that the donors detected in RE doped material are post-implantation defects that could not be annealed out in a standard annealing process [13].

Doping of Si with Er results in a characteristic photoluminescence at $1.54 \mu \mathrm{m}$ due to the optical transition between the lowest multiplets of $\mathbf{E r}^{3+}$ (see Fig. 1). Unfortunately, the efficiency of this luminescence is fairly low and strongly depends on temperature $[17,18]$. Er intrashell luminescence can be excited by tuning the

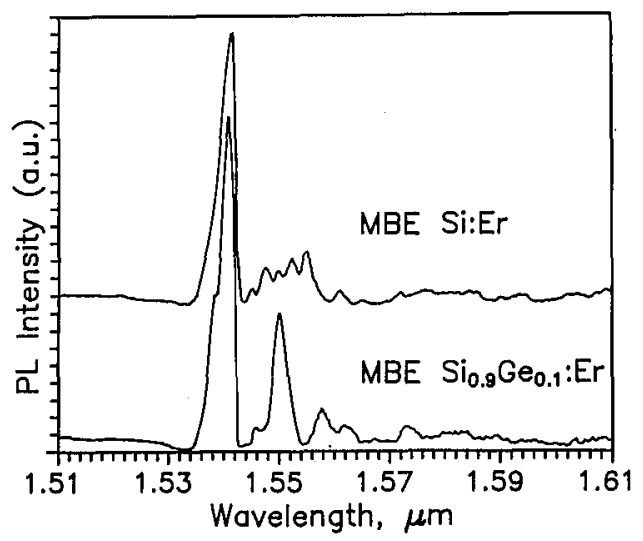

Fig. 1. Photoluminescence from erbium doped MBE Si and MBE Sio.9 $\mathrm{Ge}_{0.1}$, both grown with a substrate temperature of $500^{\circ} \mathrm{C}$. The measurement temperature was $4 \mathrm{~K}$.

photoexcitation into the resonance with the $4 f$ Er energy structure, or more efficiently by electron-hole pair creation via band-band transitions. It is also possible to obtain electroluminescence in forward biased $p-n$ junctions with the active junction region doped with REs [19]. It is obvious that an energy transfer either from photocreated or injected electron-hole pairs is responsible for generation of the Er emission. A detailed path of the transfer is not clear, however.

There is one very intricate aspect of RE emission in Si. It was found that Er emission is much more efficient in Czochralski (CZ) grown Si than in a float-zone material (FZ) [20]. In the former, concentration of oxygen is much larger than in the latter. Further experiments on co-implantation of $\mathrm{Si}$ :Er with $\mathrm{O}$ and other light impurities proved a dramatic increase in the emission efficiency and a shift of the onset of thermal quenching to much higher temperature [21]. It is well known that co-doping of RE in II-VI compounds is prerequisite for their optical activity [22]. Moreover, photoluminescence efficiency of the $\mathrm{RE}$ complexes in ionic hosts is usually the largest. It is thus quite tempting to conclude that the observed dramatic increase in the luminescence efficiency is due to the high probability of energy transfer in RE complexes with oxygen and other dopants. However, as pointed out above, the excitation of RE emission is a two-step process. The first step is a generation of electron-hole pairs that is followed by the energy transfer either to RE alone or to the RE complex. An overall efficiency must therefore 
depend critically on nonradiative processes shortening the lifetime of minority carriers. Our recent data on the temperature evolution of Er emission in MBE grown Si:Er implanted with oxygen and then annealed indicate that the presence of oxygen results in shunting the nonradiative paths for an annihilation of the photogenerated electrons and holes [17].

Optically active $\mathrm{Er}$ in Si occurs in various symmetry environments as the fine structure of the emission spectrum depends on the doping level and co-implantation as well as annealing $[17,21]$. The structure of the spectrum is, however, not sufficient to draw any detailed conclusion about the local symmetry of the Er emitting centres. Such analysis has only been done in case of InP:Er, where the Zeeman pattern indicates a cubic symmetry of the Er centre [23].

Tentative symmetry analysis in case of $\mathrm{Er}$ doped $\mathrm{Si}$ was done by the RBS/channelling measurements [24]. As expected, it was found that most of $\mathrm{Er}$ atoms are in low-symmetry sites most likely interstitial. There is no guarantee, however, that the optically active centres are the same as seen in RBS, especially as the minimum impurity concentration for the RBS analysis is close to the precipitation limit.

It can be then concluded that doping of $\mathrm{Si}$ with $\mathrm{Er}$ is quite promising for light generation from $\mathrm{Si}$, especially for application in optical communication. The efficiency is much too low, however, to speculate about high-power LEDs or lasers based on the light generation from REs in Si. Despite significant research efforts, this area is still in its infancy and it can be safely predicted that a recent wave of interest in Si doped with REs will continue for some time.

\section{RE doping of III-V compound semiconductors}

\subsection{Ytterbium in III-V semiconductors}

\subsection{1. $Y b$ in InP-based compounds}

Among the RE ions investigated in III-V compounds only $\mathrm{Yb}$ in InP behaves in a regular way. In particular, its luminescence properties are independent of the growth and doping techniques $[25,26]$. This suggests that the $\mathrm{Yb}$ atoms occupy only one type of lattice site in InP. An analysis of the Zeeman splittings of the $\mathrm{Yb}$ luminescence lines revealed local $T_{d}$ symmetry around the $\mathrm{Yb}$ ion, indicating its most probable substitutional location - $\mathrm{Yb}_{\mathbf{I n}}$ [23]. This conclusion has been confirmed by the RBS measurements [27] performed on InP samples implanted with the $150 \mathrm{keV} \mathrm{Yb}$ ions to a dose of $10^{15} \mathrm{~cm}^{-2}$ at $250^{\circ} \mathrm{C}$. The comparison of random and $\langle 100\rangle$ axial RBS spectra shows that even in as implanted samples approximately $50 \%$ of the implanted atoms are located at substitutional positions (Fig. 2). Further annealing did not increase the substitutional fraction of the $\mathrm{Yb}$ atoms. These results allowed one to estimate the solid solubility of ytterbium in InP for $8 \times 10^{19} \mathrm{~cm}^{-3}$ [27].

High solubility of $\mathrm{Yb}$ in InP suggested that in InP-based compounds, as GaInP and InPAs mixed crystals, the $\mathrm{Yb}$ atoms should also occupy substitutional positions. This idea has been verified on Yb-implanted GaInP epitaxial layers [27]. Random and $\langle 100\rangle$ axial $\mathrm{Yb}$ profiles show that the substitutional fraction of $\mathrm{Yb}$. 


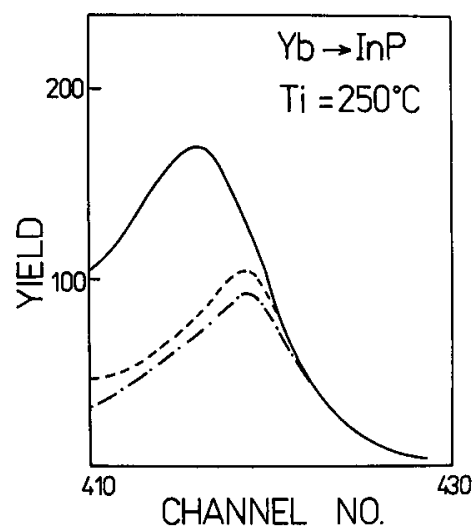

Fig. 2. $\mathrm{Yb}$ profiles in $\mathrm{InP}$ implanted at $250^{\circ} \mathrm{C}$ : random as implanted (solid line), as implanted $\langle 100\rangle$ aligned (dotted line), and $\langle 100\rangle$ aligned after $600^{\circ} \mathrm{C}$ annealing (broken line) [27].

does not exceed $15 \%$. The ytterbium solubility estimated on this basis is of the order of $3 \times 10^{19} \mathrm{~cm}^{-3}$.

The substitutional location of the $\mathrm{Yb}$ atoms in InP-based mixed crystals appeared to be a favourable condition for studying local alloy disorder in GaInP [28] and InPAs [29]. The spatially confined $4 f$-electron wave functions of the $\mathrm{Yb}$ ions ensure that only the nearest environment of an ion is sampled by its $4 f$ electrons. This offers us a unique possibility to study the local environment and bonding around the $\mathrm{Yb}$ ions in these compounds.

The $\mathrm{Yb}$ luminescence appeared to be sensitive for anionic as well as for cationic disorder in studied crystals. All the luminescence changes were ascribed to the formation of new Yb-related centres as: $\mathrm{Yb}-\mathrm{P}_{4}-\mathrm{In}_{11} \mathrm{Ga}$ in $\mathrm{GaInP}$, [28] and $\mathrm{Yb}-\mathrm{P}_{3} \mathrm{As}$ in InPAs [29], respectively. The positions of new lines and their splitting in GaInP crystal are independent of the crystal composition indicating that the strain field introduced by doping with $\mathrm{Ga}$ atoms is constant. This suggestion seems to be justified, because the nearest environment of the $\mathrm{Yb}$ atoms ( $\left.\mathrm{Yb}-\mathrm{P}_{4}\right)$ does not change in InP-GaP system, and, moreover, the bond lengths are only weakly affected by the composition of an alloy [30].

The situation in InPAs crystals is different. The line splittings due to $\langle 111\rangle$ axial strain seem also to be independent of composition, but the line positions shift slowly to lower energies, as the arsenic contents increases in the crystal. It seems that it reflects the increase in lattice constant of the alloy.

\subsection{2. $Y b$ in $G a P, G a A s$, and AlGaAs}

Ytterbium atoms behave in a very similar way in all these materials. Independent of implantation temperature they always locate in the nonsubstitutional positions (see Fig. 3). RT implantation inadvertently leads to amorphization of semiconductors. Thermal annealing was highly ineffective in restoring good crys- 
tallinity of the layers and at least $20 \%$ of damage survived the highest annealing temperatures. As a result of annealing a significant amount of the implanted atoms diffused out of the layers, and agglomerated at the surface (Fig. 4).

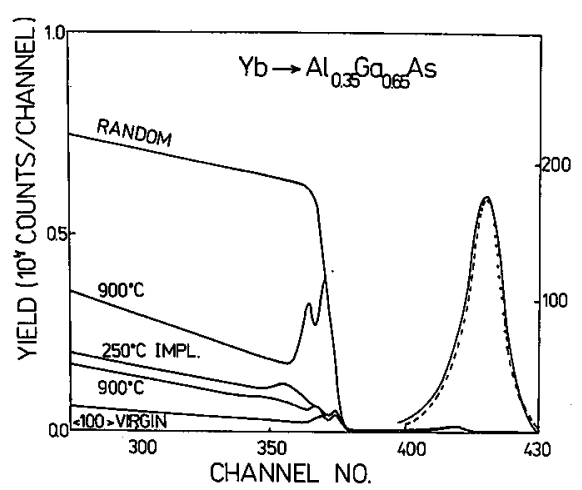

Fig. 3. RBS spectra of $\mathrm{Yb}$ implanted $\mathrm{Al}_{0.35} \mathrm{Ga}_{0.65} \mathrm{As}$. The $\mathrm{Yb}$ profiles are: random as implanted (solid line), and $\langle 100\rangle$ aligned after annealing at $900^{\circ} \mathrm{C}$ (dashed line) [27].

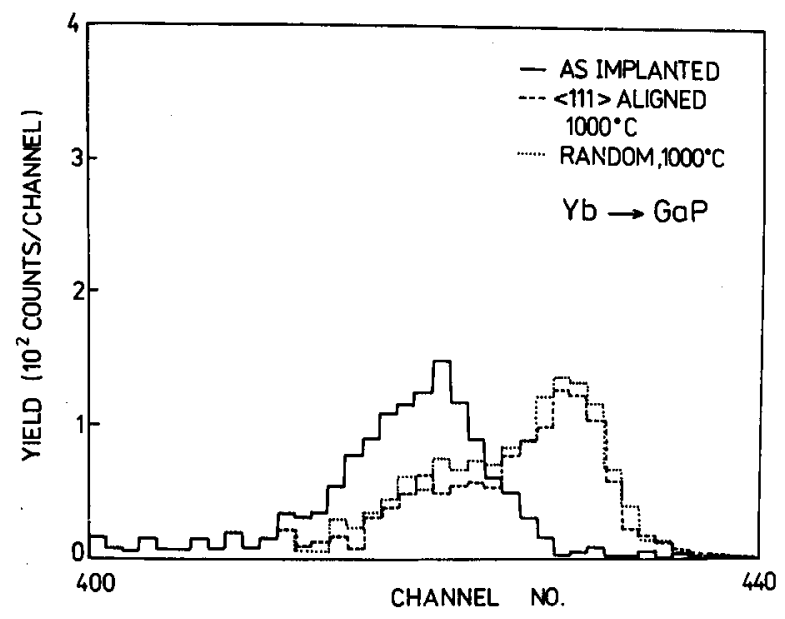

Fig. 4. Yb profiles in implanted and furnace annealed GaP [31].

Interesting behaviour of the $\mathrm{Yb}$ atoms was observed after pulse laser annealing of $\mathrm{Yb}$ implanted GaAs (Fig. 5) [31]. The ruby laser power was chosen $(0.25 \mathrm{nsec}, 0.5 \mathrm{~J} / \mathrm{cm})$ to melt all the implanted layer. The measured $\langle 100\rangle$ axial $\mathrm{Yb}$ profiles show that after the annealing the $\mathrm{Yb}$ atoms segregated completely at the surface (approximately 70\%), and at the maximum of the melting depth (the remaining $30 \%$ ). This result clearly shows that doping of $\mathrm{GaAs}$ with $\mathrm{Yb}$ during i.e. liquid phase epitaxy cannot be successful, as it was reported in [4], because the $\mathrm{Yb}$ atoms always accumulate in the melt near the growth front. 


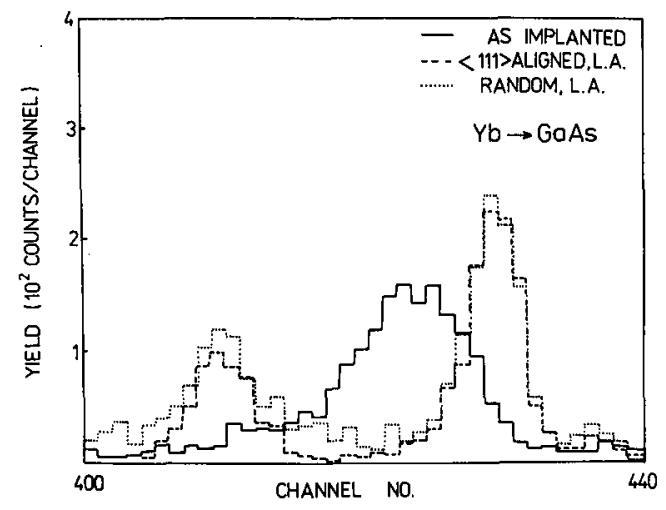

Fig. 5. Yb profiles in implanted and laser annealed GaAs [31].

\subsection{Neodymium in $G a P, G a A s$, and AlGaAs}

RBS measurements on GaP and GaAs implanted with a dose of $6 \times 10^{14} \mathrm{~cm}^{-2}$ of $150 \mathrm{keV} \mathrm{Nd}$ ions showed that the substitutional fraction of $\mathrm{Nd}$ is below sensitivity of the method. In case of AlGaAs the substitutional concentration is at the sensitivity limit of RBS and is of the order of $5 \times 10^{18} \mathrm{~cm}^{-3}$. Surprisingly, post-implantation damage in $\mathrm{AlGaAs}$ has been removed almost completely by the annealing at $900^{\circ} \mathrm{C}$ [32].

Interesting tentative results were obtained in luminescence experiments. The Nd luminescence in GaAs appeared to be independent of the disorder in the implanted layers. However, the Nd photoluminescence spectra in AlGaAs differ remarkably from those in GaAs, and their intensity is higher by a factor of four. This result suggests that $\mathrm{AlGaAs}$ :Nd could be more promising for possible optoelectronic applications.

\subsection{Erbium in GaAs}

Erbium impurity in semiconducting materials has received considerable interest, because its intra- $4 f$-shell luminescence occurring at a wavelength of $1.54 \mu \mathrm{m}$, corresponds to the minimum absorption of silica-based fibres. The data concerning luminescence of $\mathrm{Er}$ in III-V compounds are widely scattered showing either remarkable dependence on the host material, growing conditions and thermal history of the sample [33], or almost total independence of the crystalline matrix [34].

The systematic studies of lattice location of the Er atoms implanted at $250^{\circ} \mathrm{C}$ into GaAs have been done recently [35]. The erbium profiles measured for random, $\langle 100\rangle$ and $\langle 110\rangle$ axial channelling are presented in Fig. 6. The analysis of the profiles shows that the implanted Er atoms in as implanted samples are located near the middle of the $\langle 110\rangle$ channel. As a result of rapid thermal annealing (RTA) they approach the substitutional positions monotonically with increasing annealing temperature. It can be also seen that after RTA at $1000^{\circ} \mathrm{C}$ for $30 \mathrm{~s}$, the $\mathrm{Er}$ atoms 

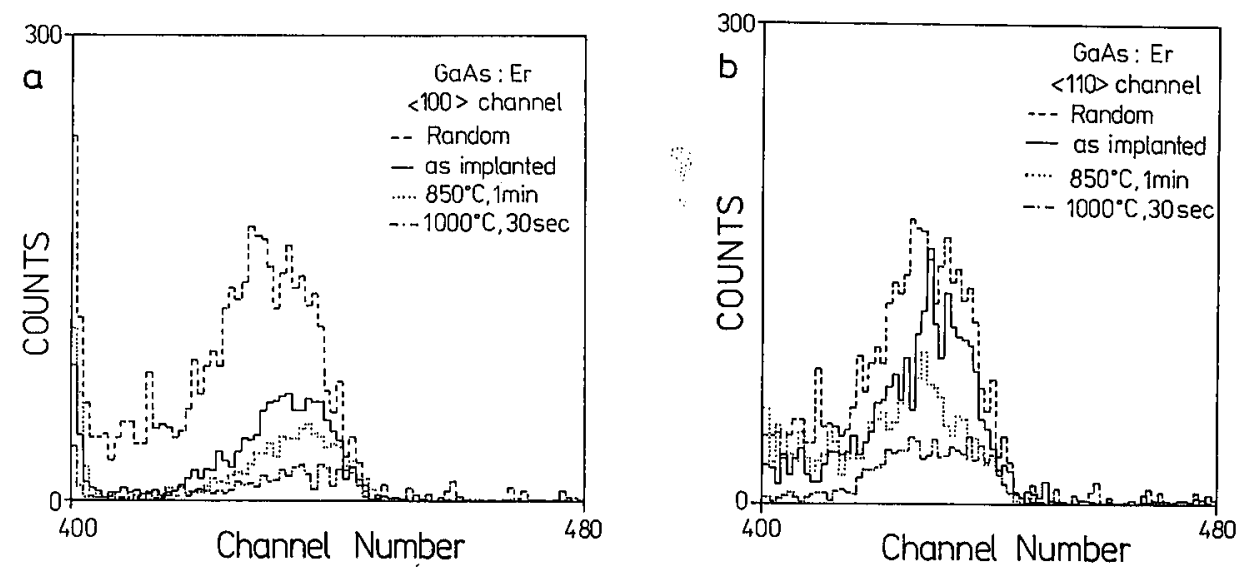

Fig. 6. Erbium profiles in implanted GaAs [35]: (a) $\langle 100\rangle$ channelling, (b) $\langle 110\rangle$ channelling.

are virtually located in the $\langle 100\rangle$ atomic rows, being only slightly displaced towards the $\langle 110\rangle$ channel.

The reason for such a location of erbium atoms can be easily explained taking into account the relatively low mismatch (1.6\%) between the lattice constants of GaAs and ErAs. Because of the different crystal structures of GaAs (zinc blende) and $\mathrm{ErAs}$ (cubic $\mathrm{NaCl}$ ), this low mismatch exists only along the $\langle 100\rangle$ direction. The Ga-As $(0.24 \mathrm{~nm})$ and $\mathrm{Er}-\mathrm{As}(0.28 \mathrm{~nm})$ bond lenghts differ markedly, therefore, the $\mathrm{Er}$ and $\mathrm{Ga}$ atoms are located along the $\langle 100\rangle$ atomic rows,but in the locations enforced by the bond lengths.

Surprisingly, a weak luminescence of Er was observed only for annealing temperatures lower than $800^{\circ} \mathrm{C}$ [35]. Optical activity of Er disappeared entirely after the Er atoms had located in the lattice sites of GaAs. This effect may be associated either with the removal of defects activating photoluminescence of $\mathrm{Er}$ or with a change of bonding character between erbium or arsenic atoms, when the Er atoms tend to occupy tetrahedral lattice sites.

Much better quality samples of Er doped III-V semiconductors are grown by MOCVD [3,36] or MBE [37, 38]. In a direct growth of doped layers all problems related to the post-implantation annealing are avoided. These techniques also allow for a growth of fairly highly doped samples without degradation of the crystal quality [36]. The onset of precipitation is similar to $\mathrm{Si}: \mathrm{Er}$ and for MBE growth it is about $10^{18} \mathrm{~cm}^{-3}$ [38]. There are two reports of RBS and channelling studies of MOCVD grown GaAs:Er [39] and MBE grown GaAs:Er and $\mathrm{Ga}_{0.5} \mathrm{Al}_{0.5} \mathrm{As:} \mathrm{Er}$ [40]. The conclusions of both reports are similar. Er in GaAs was found mostly at tetrahedral interstitial sites. For smaller Er doping substitutional positions are preferred. The emission efficiency of such samples is much lower, however. Therefore it may be concluded that only nonsubstitutional Er atoms participate in efficient light emission in GaAs. This conclusion is in agreement with that reached in our study of Er implanted GaAs [35]. 


\section{Summary}

All results reported above indicate that the doping of $\mathrm{Si}$ and III-V semiconductors with rare earths is feasible. The doped materials emit light related to the RE $4 f$-intra-shell transition. Very low solubility of REs necessitates the use of non-equilibrium doping techniques. For Si ion implantation seems to be a good choice, while for III-V semiconductors the MOCVD technology is superior to ion implantation.

The RBS and channelling measurements were done mostly on III-V semiconductors until now. The following general conclusions can be drawn from these studies.

(i) Recrystallization of the layers amorphized by room temperature implantation was retarded by the presence of RE atoms in the layers. As a result, the recrystallization was never complete with the used annealing procedures, and at least $20 \%$ of damage survived the highest-temperature annealing. The only exception of this rule is AlGaAs implanted with neodymium.

(ii) As the amorphous layers were regrowing from the crystalline substrate, most of RE atoms diffused out of the layers and accumulated at the surface. In the extreme conditions of melting of GaAs by pulse laser annealing, most of the $\mathrm{Yb}$ atoms were segregated at the surface. These results suggest that doping of III-V compounds with REs from the melt cannot be effective.

(iii) Implantation at elevated temperatures prevented amorphization of the layers. In this case migration of $\mathrm{RE}$ atoms was highly suppressed, since no redistribution of the impurity profiles was observed after annealing. It suggests that RE atoms migrate along extended defects as dislocations, grain and phase boundaries.

(iv) Solid solubility of REs in III-V compounds, taken as the substitutional concentration of atoms measured by RBS, is generally low in all the III-V:RE systems investigated. The only exception is $\mathrm{Yb}$ in InP and in InP-based ternary compounds.

(v) Optical properties of the RE atoms depend on their location in the lattice. Most clear situation has been noticed for $\mathrm{Yb}$ in InP and its ternary compounds with $\mathrm{Ga}$ [28] and As [29]. The Yb-related luminescence appeared to be very sensitive to local atomic arrangements around the impurity atoms. It offers us a unique opportunity to study local strain in semiconducting alloys.

Nonsubstitutional $\mathrm{Yb}$ atoms are optically inactive, as was shown for GaAs and AlGaAs. Very weak $\mathrm{Yb}$ luminescence in implanted GaP and GaAsP does not seem to be related to high concentration of interstitial $\mathrm{Yb}$ atoms.

Neodymium is another RE impurity whose luminescence is dependent on the crystalline matrix. Its exceptional behaviour in AIGaAs lattice in which almost perfect recrystallization of the implanted layer occurs, allows for speculations on possible advantages of this system for future applications.

(vi) Erbium atoms locate substitutionally in GaAs after annealing at $1000^{\circ} \mathrm{C}$, but at the same time they loose their optical activity. Photoluminescence of Er was observed only when the Er atoms were pushed from the lattice sites into the $\langle 110\rangle$ channel. This unexpected feature suggests that some defects or other impurities may be necessary to activate the erbium atoms. This suggests that 
nonsubstitutional erbium would disturb seriously the quality of Er-doped GaAs layers grown by MBE (usually at $550-650^{\circ} \mathrm{C}$ ).

\section{Acknowledgment}

Part of this work has been funded in U.K. by SERC.

\section{References}

[1] L.C. Kimerling, MRS Bull. 16, 42 (1991).

[2] I. Hayashi, in: Solid State Devices and Materials, Extended Abstracts of the 1992 Intern. Conf. Solid State Dev. Mat., Tsukuba 1992, Business Center for Academic Societies, Japan, Tokyo 1992, p. 10.

[3] K. Takahei, A. Taguchi, Mater. Sci. Forum 83-87, 641 (1992).

[4] J. Raczyńska, K. Fronc, J.M. Langer, A. Stappor, Appl. Phys. Lett. 53, 761 (1988).

[5] J.R. Waldrop, Appl. Phys. Lett. 46, 864 (1985).

[6] J.F. Ziegler, J.P. Biersack, U. Littmark, The Stopping and Range of Ions in Solids, Pergamon Press, Oxford 1985.

[7] S.J. Pearton, J.M. Poate, F. Sette, J.M. Gibson, D.C. Jacobson, J.S. Williams, Nucl. Instrum. Methods Phys. Res. B 19/20, 369 (1987).

[8] L.C. Feldman, J.W. Mayer, S.T. Picraux, Materials Analysis by Ion Channelling, Academic Press, New York 1982.

[9] H.C. Casey Jr., G.L. Pearson, J. Appl. Phys. 35, 3401 (1964).

[10] X. Zhao, K. Hirakawa, T. Ikoma, Appl. Phys. Lett. 54, 712 (1989).

[11] G. Jasiołek, J. Raczyńska, J. Górecka, J. Cryst. Growth 78, 105 (1986).

[12] D.J. Eaglesham, J. Michel, E.A. Fitzgerald, D.C. Jacobson, J.M. Poate, J.L. Benton, A. Polman, Y.A. Xie, L.C. Kimerling, Appl. Phys. Lett. 58, 2797 (1991).

[13] H. Efeoglu, J.H. Evans, J.M. Langer, A.R. Peaker, N.L. Rowell, J.-P. Noel, D.D. Perovic, T.E. Jackman,D.C. Houghton, in: Silicon MBE Symposium, Anaheim (Germany) 1991, MRS Proceedings, Vol. 220, 1992, in print.

[14] Ch. Delerue, M. Lannoo, Phys. Rev. Lett. 67, 3006 (1991).

[15] F.P. Widdershoven, J.P.M. Naus, Mater. Sci. Eng. B 4, 71 (1989).

[16] J.L. Benton, J. Michel, L.C. Kimerling, D.C. Jacobson, Y.-II. Xie, D.J. Eaglesham, E.A. Fitzgerald, J.M. Poate, J. Appl. Phys. 70, 2667 (1991).

[17] H. Efeoglu, J.H. Evans, T.E. Jackman, B. Hamilton; D.C. Houghton, J.M. Langer, A.R. Peaker, D.D. Perovic, I. Poole, N.L. Rowell, Semicond. Sci. Technol., 1993, in print.

[18] J. Michel, L.C. Kimerling, J.L. Benton, D.J. Eaglesham, E.A. Fitzgerald, D.C. Jacobson, J.M. Poate, Y.-H. Xie, R.F. Ferrante, Mater. Sci. Forum 83-87, 653 (1992).

[19] H. Ennen, G. Pomrenke, A. Axman, K. Eisele, W. Itaydl, J. Schneider, Appl. Phys. Lett. 46, 381 (1985).

[20] P.N. Favennec, H. L'Haridon, D. Moutonnet, M. Salvi, M. Gauneau, Jpn. J. Appl. Phys. 29, L524 (1990).

[21] J. Michel, J.L. Benton, R.F. Ferrante, D.C. Jacobson, D.J. Eaglesham, E.A. Fitzgerald, Y.-H. Xie, J.M. Poate, L.C. Kimerling, J. Appl. Phys. 70, 2672 (1981). 
[22] R. Boyn, Phys. Status Solidi B 148, 11 (1988).

[23] G. Aszodi, J. Weber, Ch. Uhleihn, L. Pu-lin, H. Ennen, U. Kaufmann, J. Schneider, J. Windscheiff, Phys. Rev. B 31, 7767 (1985).

[24] Y.S. Tang, J. Zhang, K.C. Heasman, B.J. Sealy, Solid State Commun. 72, 991 (1989).

[25] H. Ennen, U. Kaufmann, G. Pomrenke, J. Schneider, J. Windscheiff, A. Axmann, J. Cryst. Growth 64, 165 (1983).

[26] K. Uwai, H. Nakagome, K. Takahei, Appl. Phys. Lett. 50, 977 (1987).

[27] A. Kozanecki, R. Groetzschel, J. Appl. Phys. 68, 517 (1990).

[28] A. Kozanecki, Z. Kaliński, J. Raczyńska, J.M. Langer, J. Appl. Phys. 66, 3202 (1989).

[29] A. Kozanecki, Z. Kaliński, in: Proc. 20th Internat. Conf. on the Physics of Semiconductors, Thessaloniki (Greece) 1990, Eds. E.M. Anastassakis, J.M. Joannopoulos, World Scientific, Singapore 1990, p. 1989.

[30] J.C. Mikkelsen Jr., J.B. Boyce, Phys. Rev. B 28, 7130 (1983).

[31] A. Kozanecki, R. Groetzschel, J. Appl. Phys. 64, 3315 (1988).

[32] A. Kozanecki, R. Groetzschel, J. Appl. Phys. 69, 1300 (1991).

[33] K. Uwai, H. Nakagome, K. Takahei, Appl. Phys. Lett. 51, 1010 (1987).

[34] X. Zhao, K. Kirakawa, T. Ikoma, in: Gallium Arsenide and Related Compounds, Inst. Phys. Conf. Ser. No. 96, p. 389.

[35] A. Kozanecki, M. Chan, C. Jeynes, B. Sealy, K. Homewood, Solid State Commun. 78, 763 (1991).

[36] K. Uwai, H. Nakagome, K. Takahei, J. Cryst. Growth 93, 583 (1988).

[37] R.S. Smith, H.D. Muller, H. Ennen, P. Wennekers, M. Maier, Appl. Phys. Lett. 50, 49 (1987).

[38] I. Poole, K.E. Singer, A.R. Peaker, A.C. Wright, J. Cryst. Growth 121, 121 (1992).

[39] J. Nakata, M. Taniguchi, K. Takahei, Appl. Phys. Lett., 1992, to be published.

[40] E. Alves, M.F. da Silva, K.R. Evans, C.R. Jones, A.A. Melo, J. Soares, Nucl. Instrum. Methods Phys. Res. B 1993, in print. 